\title{
All-side SiNx passivated mc-Si solar cells evaluated with respect to parasitic shunting
}

\author{
I. Cesar $^{1}$, E. Bende ${ }^{1}$, G. Galbiati ${ }^{1}$, L. Janßen ${ }^{2}$, A .Weeber ${ }^{1}$, J.H. Bultman ${ }^{1}$ \\ 1) ECN Solar Energy, P.O. Box 1, 1755 ZG Petten, The Netherlands, corresponding author: cesar@ecn.nl \\ 2) Solland Solar Cells BV, Bohr 10, NL 6422 RL Heerlen, The Netherlands
}

\begin{abstract}
In search of solar cell concepts that allow processing thinner wafers ( $<150$ micron), the conventional full Al rear side is replaced by an open rear metallization combined with a dielectric passivation layer. We show a gain of $2.1 \%$ (relative) in the product of $\mathrm{Jsc} \times \mathrm{Voc}$, when we apply a passivated SiNx dielectric layer and local Al contacts on the rear of $\mathrm{p}$ type mc-Si solar cells instead of a full Al-BSF. To achieve this gain, metallization designs of $\mathrm{H}$-patterns and point-contacts were used with a rear coverage less than $8 \%$. The gain in $\mathrm{Jsc} \times \mathrm{Voc}$ is an improvement over our previously reported results for open rear side cells with rear coverage of $14 \%$. In addition, we propose a new method to quantify parasitic shunting to evaluate the efficiency potential of this cell concept. Experimental evidence shows that the parasitic shunting is one of the main limiting factors and the new method predicts a gain in Jsc and Voc in absence of this phenomenon. Omitting additional resistive losses of the open rear side cell compared to its full-coverage counterpart, we predict a gain in efficiency of about $0.6 \%$ absolute if parasitic shunting could be eliminated
\end{abstract}

\section{Introduction}

Intensive research is dedicated to reduce solar cell cost by decreasing silicon consumption through thinner wafers. As this development progressed, both the bulk diffusion length of minority carriers as well as the penetration depth of the IR part of the solar spectrum has become larger than the wafer thickness. As a direct consequence, the recombination of the minority carriers at the rear surface of the solar cell becomes more important and limits the efficiency of thin mc-Si solar cells. The most prevalent rear passivation design consists of an Al back surface field formed under the base metal contacts [1-7]. The Al backsurface-field (Al-BSF) is often formed below a non-porous$\mathrm{Si}$ alloy (eutectic) layer that crystallizes between the porous paste matrix and the BSF [8]. The thermal coefficient of the compact alloy layer differs from the one of silicon which causes thin wafers to bend resulting in additional cell breakage during processing and module assembly. Alternative passivation designs could decrease rear-side surface recombination velocities (SRV), improve the rear IR reflections and alleviate the problem of wafer bending. These bifacial cell designs consist of an open metallization pattern which contacts the cell's rear side while the open area is passivated with a dielectric layer [16]. The passivation mechanism of these dielectric layers is to neutralize recombination centers at the Si wafer surface (chemical passivation) or to electro-statically repel one type of the recombining carriers from the surface (fieldeffect passivation). The electrostatic field is induced by negative or positive fixed charges in the dielectric layer near the Si interface. For a p-type wafer, negative fixed charges repel minority carriers (i.e. electrons) from the surface in analogy to a traditional Aluminum back surface field. On the other hand, for positive fixed charges and a p-type wafer, the charge density needs to be high enough to form an (n-type) inversion layer. The formation of the inversion layer results effectively in a $p-n$ junction, typically located 10 to $100 \mathrm{~nm}$ below the interface. Usually both field effect and chemical passivation occur simultaneously. In the photovoltaic research field, various layers have been investigated like single layers or stacks of $\mathrm{SiO}_{2}$ [9], SiNx [2,10,11], and more recently $\mathrm{Al}_{2} \mathrm{O}_{3}$ [12]. Thermally grown $\mathrm{SiO}_{2}$ is known for its superior chemical passivation,
SiNx and $\mathrm{Al}_{2} \mathrm{O}_{3}$ posses high densities of positive and negative fixed charges respectively and passivate mainly due to the field-effect $[11,12]$.

We have previously reported on the development of the passivation properties of SiNx based open rear side designs with $\mathrm{H}$-patterned aluminum rear contacts (PASHA, Passivated on all side $\underline{H}$ pattern) prepared by screen printing of the firing-through type pastes. Our SiNx coatings have demonstrated SRVs as low as $10 \mathrm{~cm} / \mathrm{s}$ (on test structures) and have shown to reflect more IR light back into the solar cell compared to the full area Al-BSF, in presence of an external rear reflector. In our previous report [10] we presented an improved Al-BSF recipe and benchmarked our best performing PASHA cell at a metallization fraction of $14 \%$. As the PASHA cell underperformed in terms of $\mathrm{Jsc} \times \mathrm{Voc}$, we now report on two open rear-side designs with metal fractions below $8 \%$ which outperforms the reference cell in terms Jsc $\times$ Voc.

\section{Parasitic shunting}

Although, the lifetime of double side SiNx passivated mono crystalline wafers resulted in surface recombination values of around $10 \mathrm{~cm} / \mathrm{s}$ [2], on cell level much higher SRV values $(>500 \mathrm{~cm} / \mathrm{s})$ were fitted with PC1D. A reason why the cell performance lags behind can be explained by parasitic shunting $[11,13,14]$. This term is often evoked in literature in correlation with floating junctions that partially contact metal leads. The floating junction in the case of $\mathrm{SiNx}$ passivation is formed by fixed positive charges inducing a p-n junction near the Si/SiNx interface. Through electric conduction, electrons in the n-type inversion layer can reach contacts over a much larger length scale than the diffusion length in the p-type region normally would allow. Once the inversion layer is (partially) connected with the rear base metal contacts, large parasitic currents (up to $3.5 \mathrm{~mA} / \mathrm{cm}^{2}$ [11]) reduce the short-circuit current of the solar cell. This current path is regarded as a shunt between the inversion layer and rear metal contacts and is referred to as parasitic shunt. Like in any junction, if the voltage across this rear junction is small, a large fraction of the current flows through the shunt resistance. However, if the voltage increases, and therefore the bands flatten, the 
current that flows across the rear diode into the base of the cell, outweighs the shunt current. In this case a larger fraction of the electrons in the base is collected by the emitter and hence the rear SRV becomes effectively lower. As a consequence the effect of the parasitic shunt decreases as the voltage across the rear junction is increased by means of external bias potential (in darkness) and / or by bias illumination. This results in a red response that increases with increasing illumination intensity and therefore the Jsc-to-bias ratio is not constant but increases with increasing bias.

From the physics described, four main identifiers for parasitic shunting can be summarized [11]: 1) A strong decrease in Jsc. 2) Bias dependent response of the red part of the IQE spectrum. 3) Decreased quantum conversion efficiencies surrounding the base metal contacts, ranging over a distance larger that the minority carrier diffusion length. 4) Anomalies in the dark IV curve [14]. In this work we focus on the first three identifiers
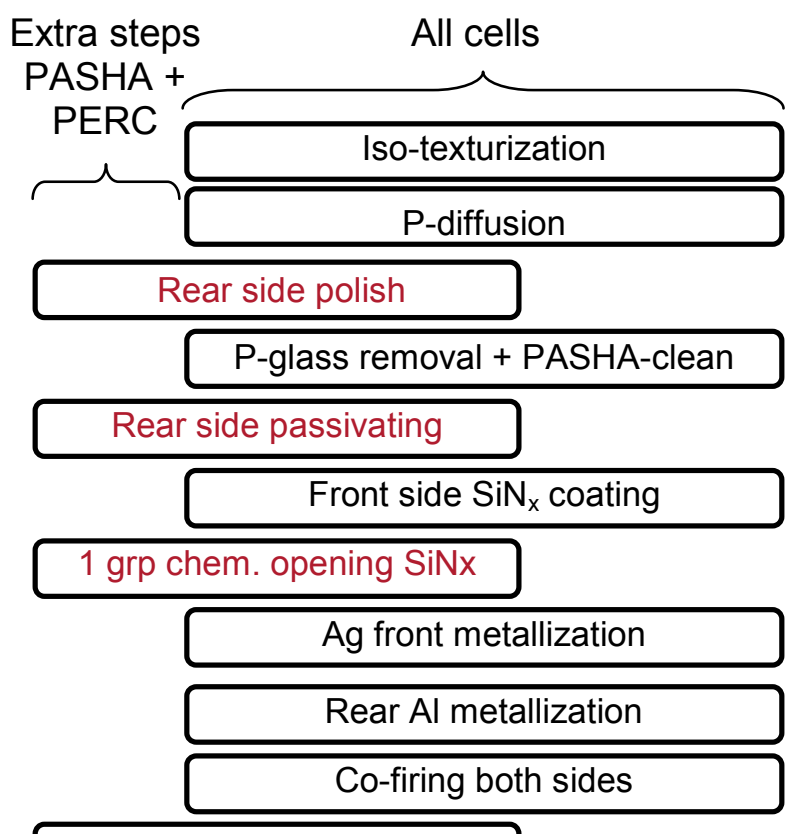

Full silver print + drying $<400 \mathrm{C}$

Figure 1, process flow of $\mathrm{Al}$ reference, PASHA and PERC processing.

\section{Experimental section}

Cell preparation and cell characterization

All solar cells are prepared with 180 micron thick p-type silicon wafer $\left(1.1-1.4 \quad \mathrm{Ohm} \cdot \mathrm{cm}, 156 \times 156 \mathrm{~mm}^{2}\right)$. The preparation method of the double side passivated solar cells as well as the full Al-BSF reference cells investigated in this work, are identical to that of our previous report with the exception of the rear metallization design [10]. The process flow is illustrated in figure 1.

It is important to mention that in this work the same extensive PASHA-clean was used on all groups as this clean was responsible for an improved response of the full
Al-BSF reference group [10]. The metallization designs consist of an $\mathrm{H}$-pattern with a $4.16 \mathrm{~mm}$ finger pitch and dot patterns consisting of 1 and $2 \mathrm{~mm}$ pitched circular dots with diameters of 220 and 550 micron, respectively. The $\mathrm{H}$-pattern is referred to as PASHA-type solar cell and contacted $8 \%$ of the rear surface while the dots are referred to as PERC type solar cells (Passivated emitter and rear cell) and contacted between $5-6 \%$ of the rear side. The designs are schematically represented in figure 2. Front and rear contacts are prepared by screen printing silver or aluminum based firing-through pastes. In the case that the aluminum paste was printed directly on the wafer, the SiNx was locally opened with a screen printed etching paste prior to printing the metal contact. As due to similar sized openings and Al-dots a misalignment was observed between the openings and the printed contacts which were unintended. This resulted in strips on one side of the contacts of several tens of microns in width, where the bare Si wafer was exposed. All open rear side patterns of both PASHA and PERC received a full area silver print in order to contact all dots and lines and to ensure a fill factor above $70 \%$. This FF criterion was used to guarantee that the Jsc would not be impeded by high resistive losses.

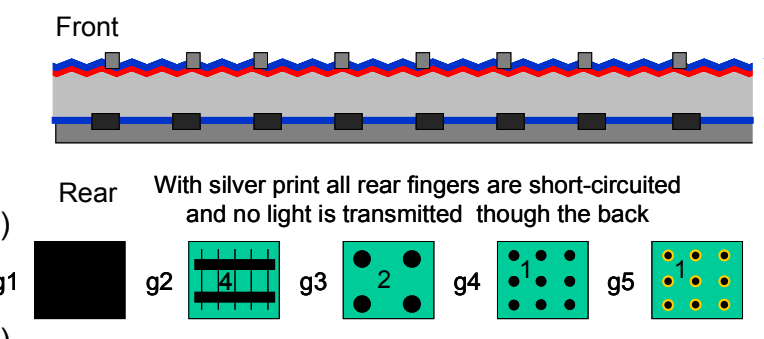

Figure 2a, Open cell concept with metal print on rear. Top down: Ag contacts, SiN coating front, texture, emitter, p-type bulk, SiNx passivating coating rear (blue) + Al-metal contacts (black), metal rear print. Figure 2b:, experimental groups consist of 5 rear designs.Gr1: Full-Al-BSF(reference); $\mathrm{Gr} 2$ fingers with $4 \mathrm{~mm}$ pitch PASHA type cell; Gr3, point contacts with $2 \mathrm{~mm}$ pitch (PERC), Gr4 with $1 \mathrm{~mm}$ pitch and $\mathrm{Gr} 5$ point contacts opened SiNx with $1 \mathrm{~mm}$ pitch (PERC). Gr2, 3 and 4: rear contact fired through SiNx layer. For group 2 to 4 , the metallization fraction on the rear of the cells that contacts the silicon ranges between 5 and $8 \%$.

The IV curves of all cells were measured under 1 sun illumination using a class A solar simulator. The spectral response is determined from a double beam setup consisting of a white bias light beam that was varied between 0 and 0.3 Sun and a chopped $(40 \mathrm{~Hz})$ monochromatic beam obtained from a $1000 \mathrm{~W}$ Xenon lamp in combination with a set of interference filters. Both beams illuminate the total area $(156 \times 156 \mathrm{~mm})$ of the cell and a lock-in amplifier is used to filter the $A C$ current. The EQE is determined relative to a calibrated reference cell. The influence of an external reflector on the spectral response was evaluated for PASHA cells by measuring with and without black absorber foil behind the solar cell in absence of the silver print. The onset of the additionally reflected light in the IQE spectrum as measured through the front of the solar cell was $1000 \mathrm{~nm}$. Also the effect of applying the silver print was measured, which resulted in a 
change of IQE above $1000 \mathrm{~nm}$ and is thus attributed to only reflection differences on the rear.

Reflection spectra were measured using an integrating sphere with a $3 \mathrm{~cm}$ circular aperture. The average of 4 reflection spectra, measured on different location on each sample, was used to calculate IQE spectra. The same rear reflector condition was used as in the spectral response measurement (i.e. black absorber or rear silver print).

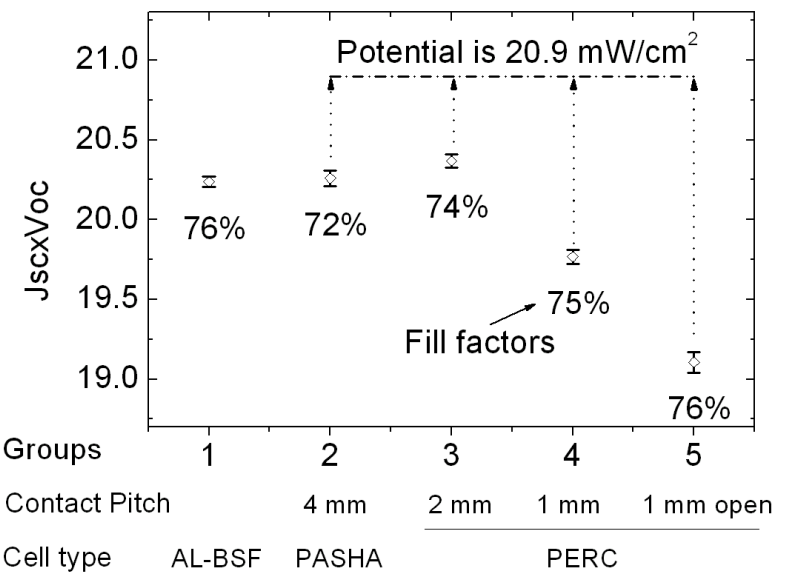

Figure 3: Product of Jsc and Voc of PASHA and PERC type solar cells in comparison to a full AL-BSF reference group. Group 1 is the control group with full Al-BSF. Group 2 to 5 are the experimental groups with various open rear metallization designs as illustrated in figure 2. Average fill factors are also shown. The potential gain in absence of parasitic shunting for group 2 to 5 is marked (dashed).

\section{White-light biased LBIC measurements and calculation}

LBIC measurements were performed under illumination of a white light bias beam obtained from a Xenon lamp. The intensity of the bias light was estimated to be 0.2 . The size of the bias spot was chosen such that it exceeded the diffusion length of the minority carriers by several path lengths. Higher bias intensities could not be applied because it resulted in a break down of the monochromatic current signal as measured by the Semilab tool. The induced laser current at $978 \mathrm{~nm}$ was about 100 microA with a specified laser spot of 100 micron in diameter.

Dedicated samples were prepared with rear fingers printed perpendicularly to those of the front in order to scan the IQE between and on the rear fingers without hindrance of the front fingers. Four different lasers were used to scan the cells: $405 \mathrm{~nm}, 863 \mathrm{~nm}, 952 \mathrm{~nm}$ and $976 \mathrm{~nm}$.

\section{Cell results}

Whereas in a previous study [10] a PASHA cell with $14 \%$ metallization showed a Jsc $\times$ Voc that was at best $1.5 \%$ lower than the full area Al-BSF cell, our best result now yields an increase of $2.2 \%$ for a PERC type cell with $2 \mathrm{~mm}$ pitched dots ( $8 \%$ metallization) compared to the full-area Al-BSF. The Jsc $\times$ Voc results are illustrated in figure 3 . It should be noted that neighboring cells of the reference group that did not receive the PASHA-clean (processed in a parallel experiment), did show a similar drop in Jsc $\times$ Voc

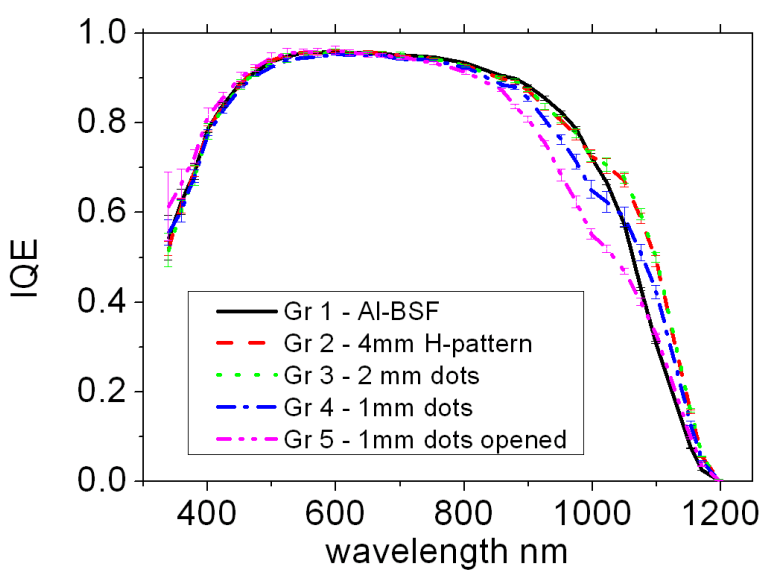

Figure 4: Average IQE spectra of PASHA and PERC type solar cells in comparison to a full AL-BSF reference group. Error bars indicate $95 \%$ confidence range of the average.

with respect to group 1 as demonstrated in our previous report [10]. This drop confirms the effect of the PASHA clean and cannot be attributed to differences in rear side surface passivation. Interestingly, cells with low metallization fraction and a $1 \mathrm{~mm}$ pitch (group 4 and 5) did result in a lower performance than group 1 , our reference.

As illustrated by the IQE spectra in figure 4, the lower Jsc $\times$ Voc values are attributed to a poor red response given the fact that no significant changes in reflection are observed (i.e. EQE spectra show same trend) and that the blue IQE responses overlap. In figure 5, the different plots of the IQE spectra relative to the full Al-BSF cells, confirm the overlap in the blue and significant differences in the IQE above 700nm. As only the rear metallization pattern changed, it is possible to assume that the bulk lifetime in

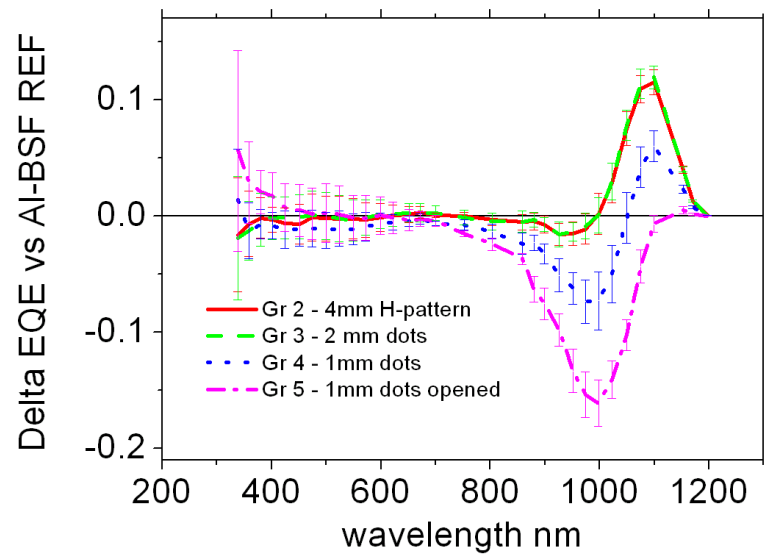

Figure 5: Average IQE spectra at $30 \%$ sun of full AL-BSF reference group substrate from the PASHA and PERC type solar cells. Error bars indicate $95 \%$ confidence range of the average.

these wafers is the same. As the rear reflection onset occurs at about $1000 \mathrm{~nm}$, the differences between groups in the wavelength range of 700 to $1000 \mathrm{~nm}$ are due to differences in effective rear surface passivation quality. Consequently, the rear passivation of the investigated cell designs measured at 0.3 sun bias light intensity can be ranked as follows from poor to best: $1 \mathrm{~mm}$ pitched opened 
dots, $1 \mathrm{~mm}$ pitched dots, $2 \mathrm{~mm}$ pitched dots and the $4 \mathrm{~mm}$ pitched $\mathrm{H}$-pattern. Integration of the difference plots multiplied with the solar spectrum confirms that the loss in current between 700 and $1000 \mathrm{~nm}$ is compensated by the gain above $1000 \mathrm{~nm}$ in the case of group 2 and 3 . The total gain in the integrated Jsc also matched well with the observed differences in the IV measurements at 1 sun which points to the fact that potential changes in passivation at 1 sun do not significantly influence Jsc.

Fitting the IQE, EQE spectra as well Jsc and Voc simultaneously with PC1D, a bulk lifetime of $21 \mu \mathrm{s}$ and a rear recombination velocity of $650 \mathrm{~cm} / \mathrm{s}$ is obtained as listed in table 1. For this fit, internal refection coefficients of $95 \%$ were used for both the front and rear side.

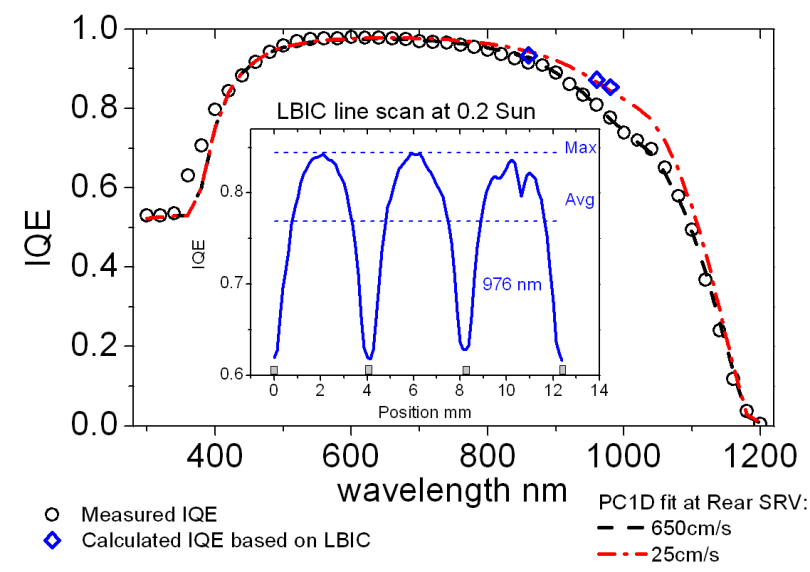

Figure 6: PC1D fits of IQE spectra with (experimental) and without (calculated) parasitic shunting. The IQE values in absence of parasitic shunting are obtained from the light biased LBIC profiles at 863,952 and $976 \mathrm{~nm}$ under $20 \%$ sun white bias light (figure 8) assuming a flat IQE profile between the rear fingers (inset). The improvement in Jsc and Voc correspond to 2.4 and $0.7 \%$ relative respectively.

\section{Discussion}

As the red response of double side SiNx passivated solar cells is dependent on the occurrence of parasitic shunting, it can be a reason for the reduction of $2.9 \%$ in $\mathrm{Jsc}^{*} \mathrm{Voc}$ for the PERC cells with $1 \mathrm{~mm}$ pitch compared to the $2 \mathrm{~mm}$ pitched contacts. A strong correlation is found between Jsc between the rear side passivated groups and the slope of the bias dependence at relatively low illumination intensities below 1 sun as illustrated in figure 7 . The larger the slope of the bias dependence of the EQE, the larger the Jsc as obtained under 1 sun. This correlation can be rationalized by a variation of the 'parasitic' shunt resistance between the inversion layer and the base contacts. The lower the shunt resistance the more light is required to charge the floating junction that will flatten the bands and re-inject electrons into the base. The reinjected electrons are now more likely collected by the front emitter and hence result in an increase of Jsc.

A plausible explanation for a change in shunt resistance is the variation in base contact spacing between the investigated metallization designs. The sheet resistance for electrons in the inversion layer increases with this distance as pointed out by Dauwe et al [11]. This fits the observation that the designs with the largest contact spacing have the steepest slopes and the higher Jsc. The fact that the bias dependency differs for the various metallization designs, while the metal coverage lies in a narrow range $(6-8 \%)$ excludes an injection-level dependent SRV of SiNx as cause of the bias-light dependent IQE.

Table 1, Fit parameters and results of PASHA type solar cell with $4.16 \mathrm{~mm}$ finger distance.

\begin{tabular}{|ll|l|l|l|}
\hline & Method & $\begin{array}{l}\text { Measured } \\
\text { IV }\end{array}$ & $\begin{array}{l}\text { Measured } \\
\text { SR and } \\
\text { RFL } \\
\text { PC1D fit }\end{array}$ & Improved \\
\hline Tau bulk & Hs & & 21 & 21 \\
$\begin{array}{l}\text { Rear SRV } \\
\text { Front }\end{array}$ & $\mathrm{cm} / \mathrm{s}$ & & 650 & 25 \\
SRV & $\mathrm{cm} / \mathrm{s}$ & & $5 \mathrm{e} 4$ & $5 \mathrm{e4}$ \\
Rint front & [-] & & 0.95 & 0.95 \\
Rint rear & [-] & & 0.95 & 0.95 \\
Jsc & $\mathrm{mA} / \mathrm{cm} 2$ & 33.8 & 33.9 & 34.7 \\
Voc & $\mathrm{mV}$ & 602 & 601 & 605 \\
\hline
\end{tabular}

The difference in shunt resistance between the two $1 \mathrm{~mm}$ pitched groups (opened en unopened $\mathrm{SiNx}$ ) is probably due to the observed misalignment of the printed aluminum dots and the SiNx openings. As a result of the misalignment a strip of tens of microns in width next to the contacts are unpassivated as no BSF nor a SiNx coating is present. This leads to an increased recombination which requires higher bias illumination intensity in order to charge the floating junction, which is visible as a slowly increasing EQE with bias light intensity in figure 7.

A direct visualization of the shunt resistance dependency on length of the shunt path, are spatial IQE maps. As the shunt resistance between the inversion layer and the contact increases away from the metal contact, an increase in conversion efficiency is expected along the same direction. This is because under equal illumination condition it is easier to charge the junction as it becomes harder for the collected electrons to migrate to the base contact were they will recombine. A spatial IQE scan performed on a cell with an $\mathrm{H}$-pattern $(4.16 \mathrm{~mm}$ finger distance) is illustrated in figure 8. For long wavelengths, that probe the rear side of the cell, a parabolic profile is obtained that ranges from one contact to the neighboring contact with a maximum in the middle. Since the typical length scale of the parabola ( $2 \mathrm{~mm}$ from contact to top) exceeds the minority carrier diffusion length ( $\sim 200$ micron) by one order of magnitude, minority carrier diffusion from the SiNx surface into the poorly passivated BSF area can be excluded as the cause of the parabolic spatial IQE shape. Omission of the white bias beam resulted in 15 to $20 \%$ lower IQE values between the rear finger (i.e. above rear SiNx) than with bias light depending of the wavelength and location. 


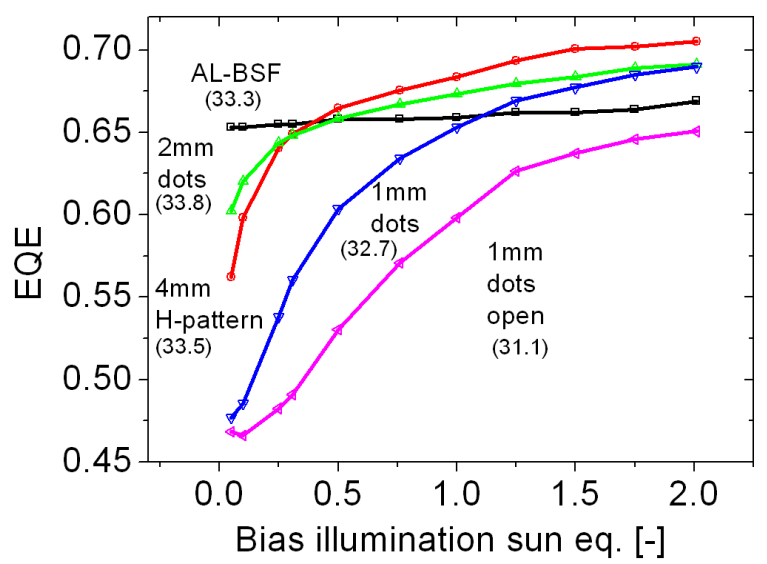

Figure 7: Bias dependence of the EQE at $998 \mathrm{~nm}$ between 0 and $200 \%$ sun obtained from all side SiNx passivated solar cell with rear metallization designs as illustrated in figure 2 . The Jsc of the presented cells is printed in parentheses.

Previously, we have stated that the efficiency of double side SiNx passivated cells was not limited by parasitic shunting [2]. This conclusion is in conflict with our current findings. The previous experiments [2] were carried out on smaller cells with all contacts fired through the SiN layers, the SR measurements were performed at $\sim 0.5$ sun bias light intensity. The parasitic shunt loss due to the pattern in these previous experiments is expected to be somewhat higher than the one of group 1 and 2. As can be seen from figure 7 the EQE is almost saturated at this injection level, and therefore the cell output will be linear, and similar to the Al reference (as was observed). However, the cells still suffer from parasitic shunting. It was discussed that opening the SiN layers and printing the contacts within the opened structure would reduce the shunt path losses. However, such a pattern will cause additional recombination losses at the bare and unpassivated $\mathrm{Si}$ surface. In the previous experiments it was observed that opening SiN layers and printing within the openings or with overlapping the SiN layers gave the same results. This means that a reduction in shunt path loss was compensated by additional recombination losses at the bare surface. Further analysis is required to determine the extent of the parasitic shunt losses for the cells with opened and unopened SiN layers.

\section{Gain potential}

In order to estimate the loss due to parasitic shunting we propose a profile in the case of an infinite shunt resistance. As a first order approximation a flat profile is assumed with a magnitude equal to the maximum of the parabola, i.e. a location independent IQE. We propose to reconstruct a new IQE spectrum for the entire cell based on this "parasitic shunt free" IQE value for the wavelengths that are available $(404,863,952$ and 976nm). As the spectral response setup illuminates the full cell, the calculated IQE values for the cell are the average of local IQE profile. This allows defining an improvement factor with which the measured IQE values for the full cell can be multiplied. The improvement factor is defined by the ratio of the maximum of the parabola and the average IQE over the selected area as illustrated in the inset of figure 6 . The calculated improvement factors range up to 1.1 for the longest wavelength of $976 \mathrm{~nm}$ and are listed in table 2 . The profile at $404 \mathrm{~nm}$ does not change because all light is absorbed in the emitter and is not affected by bulk effects. The factors are used to calculate a piece of the improved, parasitic-shunt free, IQE spectrum which is illustrated in figure 6 . In order to complete the parasitic-shunt free spectrum and to calculate the gain in Jsc and Voc, PC1D was used. First

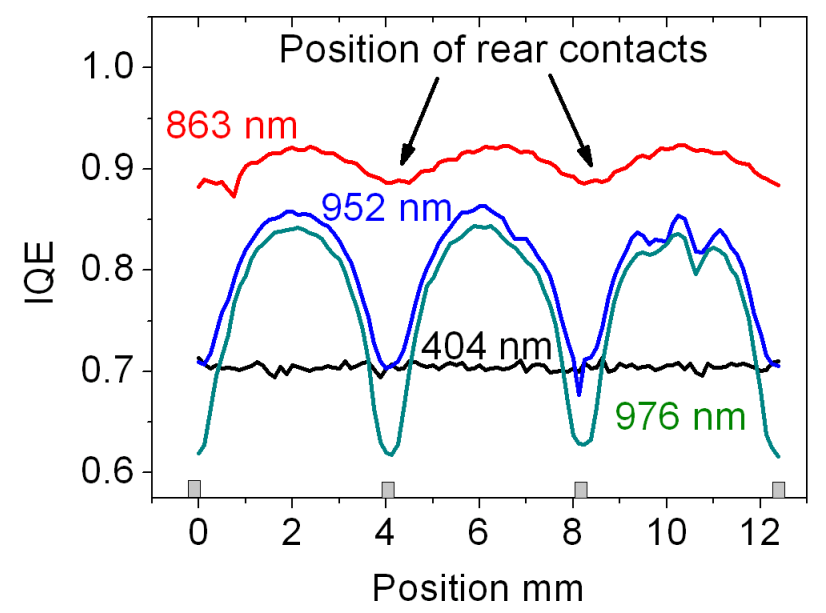

Figure 8: LBIC profiles obtained from group 2 (PASHA type cell) under front side illumination of lasers with 404, 863, 952 and $976 \mathrm{~nm}$ under $20 \%$ sun white bias light illumination (double beam experiment). The positions of the minima correspond to the rear finger pitch of $4.16 \mathrm{~mm}$ (38 fingers).

the measured IQE spectrum was fitted simultaneously with the EQE spectrum and the measured values for Jsc and Voc. The parameters used are listed in table 1. An elimination of the shunt resistance should only affect the effective SRV and therefore only this parameter was changed from 650 to $25 \mathrm{~cm} / \mathrm{s}$ to fit the four calculated IQE values. The gain in Jsc and Voc for this cell was $2.4 \%$ and $0.7 \%$, respectively. The obtained parasitic-shuntless SRV is close to the value obtained from life time measurements on mono test material. We recall that the obtained SRV is based on the first approximation of the flat profile of the parasitc shuntless spatial IQE. This approach neglects two effects that act in opposite directions. Firstly, the maximum of the parastic-shuntless spatial IQE should be higher since a higher maximum IQE is expected when two contacts would be infinitely placed way. In this work the maximum has been based on two contacts that are only separated by $4 \mathrm{~mm}$. Accounting for the higher maximum IQE will lead to an increase of the improvement factor. Secondly, since electrons that are typically within a distance of a diffusion length away from the contact have a higher probability of recombining since the contacts have a higher SRV than that of the SiNx. A parasitic-shunt free spatial IQE with rounded shoulders is therefore more accurate. If one accounts for this effect the improvement factor will be lower. Future research should quantify both counter-acting effects to obtain a more accurate improvement factor. Other uncertainties that apply to the fitted SRV value could be due to $2 \mathrm{D}$ effects of rear design 
that are lumped in the (1D) effective SRV and the exchangeability of the bulk lifetime with the rear SRV.

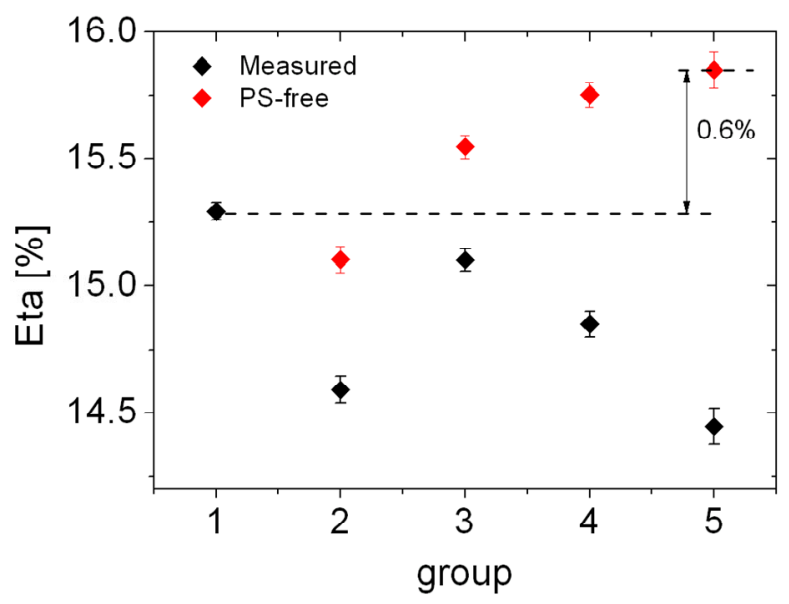

Figure 9: Measured (black) and calculated parasitic-shunt (PS) free (red) efficiency of PASHA (group 2) and PERC (group 3 to 5) type solar cells in comparison with a full Al-BSF reference (group 1).

The preceding analysis predicts a gain in Jsc $\times$ Voc of $3.1 \%$ for the $\mathrm{H}$-pattern cell if parasitic shunting can be eliminated. This gain allows predicting the absolute gain in efficiency relative to the Al-BSF reference group. The efficiency of all groups is illustrated in figure 9 . The efficiency of the Al-BSF group is the highest due to poor fill factors in group 2 and 3 and due to lower Jsc $\times$ Voc values due to parasitic shunting. The fill factor for the Al-BSF and both $1 \mathrm{~mm}$ pitched dot designs reached values between $75 \%$ to $76 \%$ while the $\mathrm{H}$-pattern and $2 \mathrm{~mm}$ pitched dot design, mainly limited by the base resistance, reached values of $72 \%$ and $74 \%$ respectively. However, if parasitic shunt could be eliminated and a gain of $3.1 \%$ in Jsc $\times$ Voc can be obtained for group 2 this $\mathrm{Jsc} \times \mathrm{Voc}$ of $20.9 \mathrm{~mW} / \mathrm{cm}^{2}$ should be attainable for all rear side passivated cells used in this experiment as the metallization fractions are similar or lower. For this assumption to hold the variation between groups in Al-BSF quality (i.e. depth and doping concentration) should be small and the recombination losses due to diffusion towards the metal contact should be limited. If this is given, the improved value of Jsc $\times$ Voc in group 2 could multiplied with the fill factor of group 5 . For a PERC type solar cell with $1 \mathrm{~mm}$ pitched dots, contacting $5 \%$ of the rear side and covered with full silver print this results in an absolute gain in efficiency of $0.55 \%$ compared to the Al-BSF reference cell.

Table 2: Calculated improvement factors for each LBIC wavelength.

\begin{tabular}{|l|l|l|l|l|}
\hline Wavelength [nm] & $\mathbf{4 0 4}$ & $\mathbf{8 6 3}$ & $\mathbf{9 5 2}$ & $\mathbf{9 7 6}$ \\
max & 71.4 & 92.4 & 86.3 & 84.4 \\
Avg & 70.4 & 90.5 & 80.0 & 76.9 \\
Improvement fact & 1.01 & 1.02 & 1.08 & 1.10 \\
\hline
\end{tabular}

It should be mentioned that for practical reasons the illumination intensity of the bias was limited to 0.2 Sun while the laser was set to 40 suns. Together with the fact that this measurement is done on a relatively small area on the wafer this exercise should be seen as a demonstration of the method rather than to focus on the absolute result. Which might be even higher as the low bias intensity underestimates the maximum of the parabola under normal solar conditions at 1 sun.

\section{Conclusion}

Open rear side p-type solar cells with a rear metallization fraction below $8 \%$, passivated by SiNx on both sides, have shown a $2.1 \%$ gain in Jsc $\times$ Voc relative to full-area Al-BSF reference cells. The gain is mainly caused by a higher external rear reflection above $980 \mathrm{~nm}$, but is limited by parasitic shunting. Parasitic shunting is the effect of increased carrier loss near the rear contacts that occur when an inversion layer at the rear surface is present. For p-type wafers, an inversion layer occurs when the rear dielectric layer contains high densities of fixed positive charges. We experimentally demonstrate parasitic shunting by bias-dependent EQE measurements and by spatial IQE measurements. We introduced a methodology to estimate cell performance if parasitic shunting could be eliminated. Based on this methodology and, moreover omitting additional resistive losses compared with the fullarea Al-BSF cell, we predict an efficiency gain of $0.6 \%$-abs for a parasitic-shunt free PERC type solar cell.

\section{References:}

[1] L. Janssen et al., Progress in Photovoltaics: Res and Appl 15, 2007, 469-475

[2] I. G. Romijn et al., Proceedings 21st EPVSEC, Dresden, September 2006, 717; Proceedings 22nd

EPVSEC, Milano, September 2007

[3] M. Hofmann et al., Proceedings 21st EPVSEC, Dresden, September 2006

[4] M. Hofmann et al., Proceedings 22nd EPVSEC,

Milano, September 2007

[5] G. Agostinelli et al., Proceedings 21st EPVSEC, Dresden, September 2006;

[6] P. Choulat et al., Proceedings 22nd EPVSEC, Milano, September 2007

[7] C. J. J. Tool et al., Solar Energy Mat. and Solar Cells 90, 2006, 3165-3173

[8] F. Huster et al, Proceedings 20nd EPVSEC, Barcelona, September 2008

[9] M. Kerr et al, Prog. Photovolt: Res. Appl. 8 (5) :529-536, 2000

[10] I. Cesar et al., Proceedings 23nd EPVSEC, Valencia, September 2008

[11] S. Dauwe, Thesis 2004

[12] B. Hoex et al, JOURNAL OF APPLIED PHYSICS 104, 044903, 2008

[13] P.P. Altermatt et al, J. Appl. Phys. 80 (6), 15 September 1996 [14] K.R. Mclntosh et al, Prog. Photovolt: Res. Appl. 7, 363- 378 (1999) 